De Tampere à Séville : bilan de la sécurité européenne $(2 / 2)$

\title{
Décision du Conseil du 28 février 2002 instituant Eurojust afin de renforcer la lutte contre les formes graves de criminalité (extraits)
}

\section{Union européenne}

\section{(2) OpenEdition \\ Journals}

\section{Édition électronique}

URL : http://journals.openedition.org/conflits/805

DOI : $10.4000 /$ conflits.805

ISSN : $1777-5345$

Éditeur :

CCLS - Centre d'études sur les conflits lilberté et sécurité, L'Harmattan

Édition imprimée

Date de publication : 1 juin 2002

ISBN : 2-7475-3030-2

ISSN : 1157-996X

Référence électronique

Union européenne, «Décision du Conseil du 28 février 2002 instituant Eurojust afin de renforcer la lutte contre les formes graves de criminalité (extraits) », Cultures \& Conflits [En ligne], 46 l été 2002, mis en ligne le, consulté le 30 mars 2021. URL : http://journals.openedition.org/conflits/805 ; DOI : https:// doi.org/10.4000/conflits.805

Ce document a été généré automatiquement le 30 mars 2021.

Creative Commons License 


\title{
Décision du Conseil du 28 février 2002 instituant Eurojust afin de renforcer la lutte contre les formes graves de criminalité (extraits)
}

\author{
Union européenne
}

1 Actes adoptés en application du titre VI du traité sur l'Union européenne (2002/187/ JAI)

2 LE CONSEIL DE L'UNION EUROPÉENNE,

3 vu le traité sur l'Union européenne, et notamment son article 31 et son article 34, paragraphe 2, point c),

4 vu l'initiative de la République fédérale d'Allemagne ainsi que celle de la République portugaise, de la République française, du Royaume de Suède et du Royaume de Belgique,

5 vu l'avis du Parlement européen,

6 considérant ce qui suit :

7 (1) Il est nécessaire d'améliorer davantage la coopération judiciaire entre les États membres, notamment dans la lutte contre les formes graves de criminalité, qui sont souvent le fait d'organisations transnationales.

8 (2) L'amélioration effective de la coopération judiciaire entre les États membres requiert d'urgence l'adoption au niveau de l'Union de mesures structurelles destinées à faciliter la coordination optimale des actions d'enquête et de poursuites des États membres couvrant le territoire de plusieurs d'entre eux, dans le respect intégral des droits et libertés fondamentaux.

9 (3) Afin de renforcer la lutte contre les formes graves de criminalité organisée, le Conseil européen de Tampere des 15 et 16 octobre 1999 a, notamment au point 46 de ses 
conclusions, décidé la création d'une unité (Euro-just) composée de procureurs, de magistrats ou d'offi-ciers de police ayant des prérogatives équivalentes.

(4) Cette unité Eurojust est instituée par la présente décision en tant qu'organe de l'Union, doté de la personnalité juridique et financé à la charge du budget général de l'Union européenne, à l'exception des salaires et émolu-ments des membres nationaux et de leurs assistants, qui sont à la charge de leurs États membres d'origine.

11 (5) Les objectifs du règlement (CE) n 10731999 du Parle-ment européen et du Conseil du 25 mai 1999 relatif aux enquêtes effectuées par l'Office européen de lutte anti-fraude (OLAF) revêtent de l'importance également en ce qui concerne Eurojust. Le collège d'Eurojust devrait adopter les mesures de mise en œuvre nécessaires pour atteindre ces objectifs. 11 devrait tenir pleinement compte des activités sensibles d'Eurojust en matière d'enquêtes et de poursuites. Dans ce cadre, il y a lieu d'exclure l'accès de l'OLAF à des documents, pièces, rapports, notes ou informations, quel qu'en soit le support, détenus ou créés dans le cadre de ces activités, qu'elles soient en cours ou clôturées, ainsi que d'interdire la transmission à l'OLAF de ces documents, pièces, rapports, notes ou informations. (6) Pour pouvoir atteindre ses objectifs de la manière la plus efficace, Eurojust devrait accomplir ses tâches soit par l'intermédiaire d'un ou plusieurs membres nationaux concernés, soit en tant que collège.

12 (7) Les autorités compétentes des États membres devraient échanger des informations avec Eurojust selon des modalités qui servent et respectent l'intérêt du fonctionnement de l'action publique.

13 (8) Les compétences d'Eurojust sont sans préjudice des compétences de la Communauté en matière de protec-tion des intérêts financiers de celle-ci et ne portent pas non plus préjudice aux conventions et accords existants, et notamment la convention européenne d'entraide judi-ciaire en matière pénale (Conseil de l'Europe) signée à Strasbourg le 20 avril 1959 ainsi que la convention relative à l'entraide judiciaire en matière pénale entre les États membres de l'Union européenne, adoptée par le Conseil le 29 mai 2000, et son protocole, adopté le 16 octobre 2001.

14 (9) Pour réaliser ses objectifs, Eurojust traite des données à caractère personnel par voie automatisée ou dans des fichiers manuels structurés. Dès lors, il convient de prendre des mesures nécessaires pour garantir un niveau de protection des données correspondant au moins à celui qui résulte de l'application des principes de la convention pour la protection des personnes à l'égard du traitement automatisé des données à caractère personnel (Conseil de l'Europe) signée à Strasbourg le 28 janvier 1981 et des modifications ultérieures, notamment le protocole ouvert à la signature le 8 novembre 2001, une fois que ces modifications seront en vigueur entre les États membres.

15 (10) Afin de contribuer à garantir et contrôler que les données a caractère personnel sont traitées correctement par Eurojust, il convient d'établir un organe de contrôle commun qui, vu la composition d'Eurojust, devrait être constitué de juges ou, si le système constitutionnel ou national le requiert, de personnes exerçant une fonction équivalente leur conférant une indépendance adéquate. Les compétences de cet organe de contrôle commun devraient être sans préjudice des compétences des tribu-naux nationaux et des recours qui peuvent être intro-duits devant ceux-ci.

16 (11) Afin d'assurer une coordination harmonieuse entre les différentes activités de l'Union et de la Communauté, et dans le respect des articles 29 et 36, paragraphe 2, du 
traité, il convient d'associer pleinement la Commission aux travaux d'Eurojust portant sur des questions géné-rales et celles qui relèvent de sa compétence. Le règle-ment intérieur d'Eurojust devrait préciser les modalités permettant à la Commission de participer aux travaux d'Eurojust dans les domaines relevant de sa compétence.

(12) Il convient de prévoir des dispositions assurant qu'Euro-just et l'Office européen de police (Europol) éta-blissent et maintiennent une coopération étroite.

(13) Il y a lieu qu'Eurojust et le Réseau judiciaire européen créé par l'action commune 98/428/JAI entretiennent des relations privilégiées. À cet effet, il convient notam-ment de placer le secrétariat du réseau au sein du secré-tariat d'Eurojust. (14) Afin de faciliter les activités d'Eurojust, il convient que les États membres puissent mettre en place ou désigner un ou plusieurs correspondants nationaux.

(15) Dans la mesure où cela est nécessaire à l'accomplisse-ment de ses tâches, il y a également lieu qu'Eurojust puisse instaurer une coopération avec des États tiers et que des accords puissent être conclus à cet effet, en priorité avec les pays candidats à l'adhésion à l'Union et d'autres pays avec lesquels des arrangements ont été convenus. (16) Étant donné que l'adoption de la présente décision demande que de nouvelles mesures législatives impor-tantes soient approuvées dans les États membres, il convient de prévoir certaines dispositions transitoires.

(17) Le point 57 des conclusions du Conseil européen de Laeken des 14 et 15 décembre 2001 prévoit que, en attendant un accord global sur le siège de certaines agences, Eurojust pourra débuter ses activités à La Haye. (18) La présente décision respecte les droits fondamentaux et observe les principes reconnus par l'article 6, paragraphe 2 , du traité et reflétés par la Charte des droits fondamen-taux de l'Union européenne,

DÉCIDE :

23 Article premier

Création et personnalité juridique

La présente décision institue une unité dénommée «Eurojust » en tant qu'organe de l'Union.

Eurojust est dotée de la personnalité juridique.

Article 2

Composition

1. Eurojust est composée d'un membre national, détaché par chaque État membre conformément à son système juridique, ayant la qualité de procureur, de juge ou d'officier de police ayant des prérogatives équivalentes.

2. Chaque membre national peut être assisté par une personne. En cas de nécessité et avec l'accord du collège visé à l'article 10, plusieurs personnes peuvent assister le membre national. Un de ces assistants peut remplacer le membre national.

Article 3

Objectifs

1. Dans le cadre des enquêtes et des poursuites concernant deux États membres ou plus et portant sur les comportements criminels visés à l'article 4 dans le domaine de la criminalité grave, notamment lorsqu'elle est organisée, les objectifs assi-gnés à Eurojust sont : 
31 a) de promouvoir et d'améliorer la coordination entre les auto-rités compétentes des États membres concernant des enquêtes et des poursuites dans les États membres, en tenant compte de toute demande émanant d'une autorité compé-tente d'un État membre et de toute information fournie par un organe compétent en vertu de dispositions arrêtées dans le cadre des traités ;

32 b) d'améliorer la coopération entre les autorités compétentes des États membres, notamment en facilitant la mise en œuvre de l'entraide judiciaire internationale et l'exécution des demandes d'extradition ;

33 c) de soutenir, par ailleurs, les autorités compétentes des États membres pour renforcer l'efficacité de leurs enquêtes et de leurs poursuites.

2. Selon les modalités prévues par la présente décision et à la demande d'une autorité compétente d'un État membre, Eurojust peut également apporter son soutien à des enquêtes ou des poursuites concernant ce seul État membre et un Etat tiers si un accord instaurant une coopération en vertu de l'article 27, paragraphe 3, a été conclu avec ledit État ou si, dans un cas particulier, il y a un intérêt essentiel à apporter ce soutien.

35 3. Selon les modalités prévues par la présente décision et à la demande soit d'une autorité compétente d'un État membre, soit de la Commission, Eurojust peut également apporter son soutien à des enquêtes ou des poursuites concernant ce seul État membre et la Communauté.

Article 4

Compétences

a) les types de criminalité et les infractions pour lesquels Europol a, à tout moment, compétence pour agir en appli-cation de l'article 2 de la convention Europol du 26 juillet 1995 ;

b) les types de criminalité suivants :

40

- la criminalité informatique,

41

- la fraude et la corruption, ainsi que toute infraction pénale touchant aux intérêts financiers de la Commu-nauté européenne,

42

- le blanchiment des produits du crime,

43

- la criminalité au détriment de l'environnement,

44

- la participation à une organisation criminelle au sens de l'action commune 98/733/JAI du Conseil du 21 décembre 1998 relative à l'incrimination de la participa-tion à une organisation criminelle dans les États membres de l'Union européenne ;

c) d'autres infractions ayant été commises en liaison avec les types de criminalité et les infractions visés aux points $a$ ) et $b$ ).

2. Pour d'autres types d'infractions que celles visées au para-graphe 1, Eurojust peut, à titre complémentaire, conformément à ses objectifs, et à la demande d'une autorité 
compétente d'un État membre, apporter son concours à des enquêtes ou à des poursuites.

47 Article 5

Tâches d'Eurojust

1. Afin de remplir ses objectifs, Eurojust accomplit ses tâches :

a) par l'intermédiaire d'un ou plusieurs des membres nationaux concernés conformément à l'article 6, ou

b) en tant que collège conformément à l'article 7 dans les cas :

i) pour lesquels un ou plusieurs membres nationaux concernés par une affaire traitée par Eurojust en font la demande, ou

ii) relatifs à des enquêtes ou des poursuites ayant une incidence au niveau de l'Union ou pouvant concerner des États membres autres que ceux directement impli-qués, ou

iii) dans lesquels une question générale relative à la réalisa-tion de ses objectifs se pose, ou

iv) prévus par d'autres dispositions de la présente décision.

2. Lorsqu'elle accomplit ses tâches, Eurojust indique si elle agit par l'intermédiaire d'un ou plusieurs membres nationaux au sens de l'article 6 ou en tant que collège au sens de l'article 7.

\section{Article 6}

Tâches d'Eurojust exercées par l'intermédiaire de ses membres nationaux

Lorsque Eurojust agit par l'intermédiaire de ses membres natio-naux concernés, elle :

a) peut demander aux autorités compétentes des États membres concernés d'envisager :

i) d'entreprendre une enquête ou des poursuites sur des faits précis ;

ii) d'accepter que l'une d'elles puisse être mieux placée pour entreprendre une enquête ou des poursuites sur des faits précis ;

iii) de réaliser une coordination entre les autorités compé-tentes des États membres concernés ;

iv) de mettre en place une équipe d'enquête commune en conformité avec les instruments de coopération perti-nents ;

v) de lui fournir toute information nécessaire pour accomplir ses tâches ;

b) assure l'information réciproque des autorités compétentes des États membres concernés sur les enquêtes et les pour-suites dont elle a connaissance ;

c) assiste, à leur demande, les autorités compétentes des États membres en vue d'assurer la meilleure coordination possible des enquêtes et des poursuites ;

d) apporte son concours afin d'améliorer la coopération entre les autorités compétentes des États membres ;

e) coopère avec le Réseau judiciaire européen et le consulte, y compris en utilisant sa base documentaire et en contribuant à l'améliorer ;

f) apporte, dans les cas visés à l'article 3, paragraphes 2 et3, et avec l'accord du collège, son soutien à des enquêtes et des poursuites concernant les autorités compétentes d'un seul Etat membre ; 
69 g) peut, conformément à ses objectifs et dans le cadre de l'article 4, paragraphe 1, afin d'améliorer la coopération et la coordination entre les autorités compétentes des États membres, transmettre des demandes d'entraide judiciaire lorsqu'elles :

i) émanent d'une autorité compétente d'un État membre,

71 ii) concernent une enquête ou une poursuite menée par cette autorité dans une affaire déterminée, et,

iii) nécessitent, en vue d'une exécution coordonnée, son intervention.

73 Article 7

Tâches d'Eurojust exercées en tant que collège

Lorsque Eurojust agit en tant que collège, elle :

a) peut, en ce qui concerne les types de criminalité et les infractions visés à l'article 4, paragraphe 1, demander, de manière motivée, aux autorités compétentes des États membres concernés :

i) d'entreprendre une enquête ou des poursuites sur des faits précis ;

77 ii) d'accepter que l'une d'elles puisse être mieux placée pour entreprendre une enquête ou des poursuites sur des faits précis ;

78 iii) de réaliser une coordination entre les autorités compé-tentes des États membres concernés ;

iv) de mettre en place une équipe d'enquête commune en conformité avec les instruments de coopération perti-nents ;

v) de lui fournir toute information nécessaire pour accomplir ses tâches ;

b) assure l'information réciproque des autorités compétentes des États membres sur les enquêtes et les poursuites dont elle a connaissance et ayant une incidence au niveau de l'Union ou qui pourraient concerner des États membres autres que ceux directement concernés ;

82 c) assiste, à leur demande, les autorités compétentes des États membres en vue d'assurer la meilleure coordination possible des enquêtes et des poursuites ;

d) apporte son concours afin d'améliorer la coopération entre les autorités compétentes des États membres, notamment sur la base de l'analyse effectuée par Europol ;

84 e) coopère avec le Réseau judiciaire européen et le consulte, y compris en utilisant sa base documentaire et en contribuant à l'améliorer ;

f) peut apporter son concours à Europol notamment en lui fournissant des avis, sur la base des analyses qu'il a effec-tuées ;

g) peut fournir un soutien logistique dans les cas visés aux points a), c) et d). Ce soutien logistique peut notamment comporter une aide pour la traduction, l'interprétation et l'organisation de réunions de coordination.

87 Article 8

Motivation

88 Si les autorités compétentes de l'État membre concerné décident de ne pas suivre la demande visée à l'article 7, point a), elles communiquent à Eurojust leur décision et les raisons qui la motivent, sauf si, dans les cas visés à l'article 7, point a) i), ii) et v), elles ne peuvent apporter une motivation dans la mesure où : 


\section{État membre pour ce qui concerne leur statut. La durée du mandat des membres} nationaux est déterminée par l'État membre d'origine. Elle est de nature à permettre un bon fonc-tionnement d'Eurojust.

93 demandes formulées dans le cadre de l'article 6, point a), passent par le membre national.

94 3. Chaque État membre définit la nature et l'étendue des pouvoirs judiciaires qu'il confère à son membre national sur son propre territoire. Il définit également le droit pour un membre national d'agir à l'égard des autorités judiciaires étran-gères, conformément aux engagements internationaux qu'il a souscrits. Au moment de la désignation du membre national, et le cas échéant à tout autre moment, l'État membre notifie à Eurojust et au secrétariat général du Conseil sa décision afin que celui-ci informe les autres États membres. Ceux-ci s'en-gagent à accepter et à reconnaître les prérogatives ainsi confé-rées dans la mesure où elles sont conformes aux engagements internationaux.

4. Afin de remplir les objectifs d'Eurojust, le membre national a accès à l'information contenue dans le casier judi-ciaire national ou dans tout autre registre de son État membre de la même manière que son droit national le prévoit pour un procureur, juge ou officier de police ayant des prérogatives équivalentes. compétentes de son État membre.

6. Dans l'exercice de ses fonctions, le membre national indique, le cas échéant, s'il agit en vertu des pouvoirs judiciaires qui lui sont conférés conformément au paragraphe 3.

\section{Article 10}

Collège

1. Le collège est composé de tous les membres nationaux. Chaque membre national dispose d'une voix.

2. Le Conseil, après consultation de l'organe de contrôle commun visé à l'article 23 pour ce qui concerne les disposi-tions relatives au traitement des données à caractère personnel, approuve le règlement intérieur d'Eurojust sur proposition du collège que celui-ci aura préalablement adoptée à l'unanimité. Les dispositions du règlement intérieur qui concernent le traite-ment des données à caractère personnel peuvent faire l'objet d'une approbation séparée par le Conseil.

101 3. Lorsqu'il agit selon l'article 7, point a), le collège statue à la majorité des deux tiers. Le collège prend ses autres décisions conformément au règlement intérieur.

Article 11

Rôle de la Commission 
103 1. La Commission est pleinement associée aux travaux d'Eu-rojust conformément à l'article 36, paragraphe 2, du traité. Elle participe, dans les domaines relevant de sa compétence, à ces travaux.

104 2. Dans le cadre des travaux d'Eurojust concernant la coor-dination des enquêtes et poursuites, la Commission peut être invitée à apporter ses connaissances spécialisées.

3. Eurojust peut convenir avec la Commission des modalités pratiques nécessaires pour renforcer leur coopération.

106 Article 12

Correspondants nationaux

107 1. Chaque État membre peut mettre en place ou désigner un ou plusieurs correspondants nationaux. Cette mise en place ou cette désignation est hautement prioritaire en matière de terro-risme. Les relations entre le correspondant national et les auto-rités compétentes des États membres sont régies par le droit national. Les correspondants nationaux ont leur lieu de travail dans l'État membre qui les a désignés.

108 2. Lorsque l'État membre désigne un correspondant national, celui-ci peut être un point de contact du Réseau judiciaire européen.

109 3. Les relations entre le membre national et le correspon-dant national n'excluent pas des relations directes entre le membre national et ses autorités compétentes.

110 Article 13

Échanges d'informations avec les États membres et entre membres nationaux

111 1. Les autorités compétentes des États membres peuvent échanger avec Eurojust toute information nécessaire en vue de l'accomplissement des tâches de celui-ci, conformément à l'article 5 .

112 2. Conformément à l'article 9, les membres nationaux d'Eu-rojust sont habilités à échanger, sans autorisation préalable, toute information nécessaire en vue de l'accomplissement de ses tâches, entre eux ou avec les autorités compétentes de leur État membre.

113 Article 14

Traitement des données à caractère personnel

114 1. Dans la mesure où cela est nécessaire pour réaliser ses objectifs, Eurojust peut, dans le cadre de ses compétences et afin de mener à bien ses tâches, traiter les données à caractère personnel, par voie automatisée ou dans des fichiers manuels structurés.

2. Eurojust prend les mesures nécessaires pour garantir un niveau de protection des données à caractère personnel corres-pondant au moins à celui qui résulte de l'application des prin-cipes de la convention du Conseil de l'Europe du 28 janvier 1981 et de ses modifications ultérieures qui seraient en vigueur entre les États membres.

116 3. Les données à caractère personnel traitées par Eurojust sont adéquates, pertinentes et non excessives au regard des fins auxquelles elles sont traitées et, compte tenu des informations fournies par les autorités compétentes des États membres ou d'autres partenaires conformément aux articles 13 et 26, elles sont également exactes et mises à jour. Les données à caractère personnel traitées par Eurojust sont traitées loyalement et licite-ment. 
117 4. Conformément à la présente décision, Eurojust établit un index des données relatives aux enquêtes et peut créer des fichiers de travail temporaires comportant également des données à caractère personnel.

Article 15

Restrictions relatives au traitement des données à carac-tère personnel

1. Lors du traitement des données conformément à l'article 14, paragraphe 1, Eurojust peut seulement traiter les données à caractère personnel ci-après concernant des personnes qui, au regard du droit national des États membres concernés, font l'objet d'une enquête ou d'une poursuite pénale pour un ou plusieurs types de criminalité et infractions définis à l'article 4 :

a) le nom de famille, le nom de jeune fille, le prénom et, le cas échéant, le nom d'emprunt ;

b) la date et le lieu de naissance ;

c) la nationalité ;

d) le sexe ;

e) le lieu de résidence, la profession et le lieu où se trouve la personne concernée ;

f) les numéros de sécurité sociale,

les informations relatives aux personnes morales, si elles comportent des informations concernant des personnes physiques identifiées ou identifiables qui font l'objet d'une enquête ou d'une poursuite légale ;

h) les comptes en banque et les comptes auprès d'autres insti-tutions financières ;

i) la description et la nature des faits reprochés, la date de leur commission, leur qualification pénale et l'état d'avancement des enquêtes ;

129 j) les faits laissant prévoir une extension de l'affaire au niveau international ;

130 k) des informations relatives à l'appartenance présumée à une organisation criminelle.

131 2. Lors du traitement des données conformément à l'article 14, paragraphe 1, Eurojust peut seulement traiter les données à caractère personnel ci-après concernant des personnes qui, au regard du droit national des États membres concernés, sont considérés comme témoins ou victimes dans une enquête ou une poursuite pénale concernant un ou plusieurs types de criminalité et infractions définis à l'article 4 :

132 a) le nom de famille, le nom de jeune fille, le prénom et, le cas échéant, le nom d'emprunt ;

b) la date et le lieu de naissance ;

c) la nationalité ;

d) le sexe ;

e) le lieu de résidence, la profession et le lieu où se trouve la personne concernée ;

f) la description et la nature des faits les concernant, la date de leur commission, leur qualification pénale et l'état d'avance-ment des enquêtes.

3. Toutefois, dans des cas exceptionnels, Eurojust peut également traiter, pendant un temps limité, d'autres données à caractère personnel relatives aux circonstances d'une infraction lorsqu'elles sont d'un intérêt immédiat pour les enquêtes en cours à la coordination desquelles Eurojust contribue et prises en compte dans ce cadre, pour 
autant que le traitement de ces données spécifiques soit conforme aux dispositions prévues aux articles 14 et 21 .

Le délégué à la protection des données visé à l'article 17 est immédiatement informé du recours au présent paragraphe.

作

2, la décision de les traiter est prise conjointement par au moins deux membres nationaux.

141 4. Les données à caractère personnel, qu'elles soient ou non l'objet d'un traitement automatisé, qui révèlent l'origine raciale ou ethnique, les opinions politiques, les convictions religieuses ou philosophiques ou l'appartenance syndicale, ainsi que celles relatives à la santé et à la vie sexuelle ne peuvent être traitées par Eurojust que si elles sont nécessaires aux enquêtes natio-nales concernées et à la coordination au sein d'Eurojust.

142 Le délégué à la protection des données est immédiatement informé du recours au présent paragraphe.

Ces données ne peuvent pas être traitées dans l'index prévu à l'article 16, paragraphe 1.

Lorsque ces autres données concernent des témoins ou victimes au sens du paragraphe 2 , la décision de les traiter est prise par le collège. (...)

Article 22

Sécurité des données

146 1. En ce qui concerne le traitement des données à caractère personnel dans le cadre de la présente décision, Eurojust et chaque État membre, dans la mesure où il est concerné par les données transmises par Eurojust, assurent la protection desdites données contre la destruction accidentelle ou illicite, la perte accidentelle ou la divulgation, la modification et l'accès non autorisés ou contre toute autre forme de traitement non auto-risé.

147 2. Le règlement intérieur contient les mesures techniques et les modalités organisationnelles nécessaires à l'exécution de la présente décision pour ce qui concerne la sécurité des données, et notamment des mesures qui sont propres à :

148 a) interdire à toute personne non autorisée d'accéder aux installations utilisées pour le traitement de données à carac-tère personnel ;

b) empêcher que des supports de données ne puissent être lus, copiés, modifiés ou enlevés par une personne non autorisée ;

150 c) empêcher l'introduction non autorisée dans le fichier ainsi que toute prise de connaissance, modification ou effacement non autorisés de données à caractère personnel intégrées ;

151 d) empêcher que des systèmes de traitement automatisé de données ne puissent être utilisés par des personnes non autorisées à l'aide d'installations de transmission de données ;

152 e) garantir que, pour l'utilisation d'un système de traitement automatisé de données, les personnes autorisées ne puissent accéder qu'aux données relevant de leur compétence ;

153 f) garantir qu'il puisse être vérifié et constaté à quelles instances des données à caractère personnel sont transmises en cas de transmission de données ; 
154 g) garantir qu'il puisse être vérifié et constaté a posteriori quelles données à caractère personnel ont été introduites dans les systèmes de traitement automatisé de données, à quel moment et par quelle personne elles y ont été intro-duites ;

h) empêcher que lors de la transmission de données à caractère personnel ainsi que lors du transport de supports de données, les données ne puissent être lues, copiées, modi-fiées ou effacées de façon non autorisée.

Article 23

Organe de contrôle commun

157 1. Il est créé un organe de contrôle commun indépendant qui contrôle, de manière collégiale, les activités d'Eurojust visées aux articles 14 à 22 afin d'assurer que les données à caractère personnel sont traitées dans le respect de la présente décision. Afin d'accomplir ces tâches, l'organe de contrôle commun est habilité à accéder sans réserves à tous les fichiers dans lesquels ces données à caractère personnel sont traitées. Eurojust fournit à l'organe de contrôle commun toutes les informations contenues dans les fichiers qu'il demande et l'as-siste dans l'exécution de ses tâches par tous les autres moyens.

158 L'organe de contrôle commun se réunit au moins une fois par semestre. En outre, il se réunit dans les 3 mois qui suivent l'introduction d'un recours et peut être convoqué par son prési-dent lorsqu'au moins deux États membres en formulent la demande.

159 En vue de constituer cet organe de contrôle commun, chaque État membre désigne, conformément à son système juridique, un juge, non-membre d'Eurojust, ou, si le système constitu-tionnel ou national le requiert, une personne exerçant une fonction lui conférant une indépendance adéquate, pour figurer sur la liste des juges, susceptibles de siéger dans l'organe de contrôle commun en qualité de membre ou de juge ad hoc. La durée de la désignation ne peut être inférieure à 18 mois. La révocation de la désignation est régie par les principes de révocation applicables en vertu du droit interne de l'État membre d'origine. La désignation et sa révocation sont notifiées au secrétariat général du Conseil et à Eurojust.

160 2. L'organe de contrôle commun est composé de 3 membres permanents et, selon ce qui est prévu au paragraphe 4 , de juges ad hoc.

161 3. Le juge désigné par un État membre devient membre permanent un an avant que son État n'exerce la présidence du Conseil et ce pour une durée d'un an et 6 mois.

162 Le juge désigné par l'État membre qui exerce la présidence du Conseil assume la présidence de l'organe de contrôle commun.

163 4. Un ou plusieurs juges ad hoc siègent également, pour la seule durée de l'examen d'un recours concernant des données à caractère personnel provenant de l'État membre qui les a dési-gnés.

164 5. La composition de l'organe de contrôle commun vaut pour l'ensemble de la durée de l'examen d'un recours même si les membres permanents ont atteint la fin de leur mandat au titre du paragraphe 3 .

165 6. Chaque membre et chaque juge ad hoc possèdent une voix délibérative. En cas de parité des voix, la voix du président est prépondérante.

166 7. L'organe de contrôle commun examine les recours qui lui sont présentés conformément à l'article 19, paragraphe 8 , et à l'article 20, paragraphe 2, effectue les contrôles conformément au paragraphe 1 , premier alinéa, du présent article. Si 
l'organe de contrôle commun estime qu'une décision prise par Eurojust ou un traitement de données effectué par lui n'est pas conforme à la présente décision, la question est renvoyée devant Eurojust qui se soumet à la décision de l'organe de contrôle commun.

8. Les décisions de l'organe de contrôle commun sont défi-nitives et contraignantes à l'égard d'Eurojust.

168 9. Les personnes désignées par les États membres conformé-ment au paragraphe 1 , troisième alinéa, présidées par le prési-dent de l'organe de contrôle commun, adoptent un règlement intérieur et de procédure, qui, pour l'examen d'un recours, prévoit des critères objectifs pour la désignation des membres de l'organe.

10. Les frais de secrétariat sont couverts par le budget d'Eu-rojust. Le secrétariat de l'organe de contrôle commun est indé-pendant dans sa fonction au sein du secrétariat d'Eurojust.

170 11. Les membres de l'organe de contrôle commun sont soumis à l'obligation de confidentialité prévue à l'article 25 .

12. L'organe de contrôle commun fait rapport une fois par an au Conseil.

172 Article 24

Responsabilité du fait d'un traitement non autorisé ou incorrect de données

1. Eurojust est responsable, conformément au droit national de l'État membre dans lequel est établi son siège, de tout dommage causé à une personne et qui résulte d'un traitement de données non autorisé ou incorrect dont il est l'auteur.

2. Les plaintes contre Eurojust dans le cadre de la responsa-bilité visée au paragraphe 1 sont introduites devant les tribu-naux de l'État membre où son siège est situé.

175 3. Tout État membre est responsable, conformément à son droit national, de tout dommage causé à une personne et qui résulte d'un traitement de données non autorisé ou incorrect dont il est l'auteur et qui ont été communiquées à Eurojust.

176 Article 25

Confidentialité

177 1. Les membres nationaux et leurs assistants visés à l'article 2, paragraphe 2, le personnel d'Eurojust et les correspondants nationaux, s'ils existent, ainsi que le délégué à la protection des données sont tenus à une obligation de confidentialité et ce sans préjudice de l'article 9, paragraphe 1.

178 2. L'obligation de confidentialité s'applique à toute personne et à tout organisme appelés à travailler avec Eurojust.

179 3. L'obligation de confidentialité demeure également après cessation des fonctions, du contrat de travail ou de l'activité des personnes visées aux paragraphes 1 et 2 .

180 4. Sans préjudice de l'article 9 , paragraphe 1 , l'obligation de confidentialité s'applique à toutes les informations que reçoit Eurojust.

181 Article 26

Relations avec les partenaires

182 1. Eurojust établit et maintient une coopération étroite avec Europol, dans la mesure où celle-ci est nécessaire à l'accomplis-sement des tâches d'Eurojust et à la réalisation de ses objectifs, et compte tenu de la nécessité d'éviter les doubles emplois inutiles. Les éléments essentiels de cette coopération sont déter-minés par un accord qui doit être 
approuvé par le Conseil, après consultation de l'organe de contrôle commun pour ce qui concerne les dispositions relatives à la protection de données.

2. Eurojust entretient avec le Réseau judiciaire européen des relations privilégiées basées sur la concertation et la complé-mentarité, notamment entre le membre national, les points de contact d'un même État membre et, lorsqu'il existe, le correspondant national. Afin de garantir une coopération efficace, les mesures ci-après sont prises :

a) Eurojust a accès aux informations recueillies au niveau central par le Réseau judiciaire européen conformément à l'article 8 de l'action commune 98/428/JAI et au réseau de télécommunications mis en place en vertu de l'article 10 de ladite action commune ;

b) par dérogation à l'article 9, paragraphe 3, de l'action commune 98/428/JAI, le secrétariat du Réseau judiciaire européen est placé au sein du secrétariat d'Eurojust. Il en forme une unité distincte et autonome sur le plan fonc-tionnel. Il bénéficie des moyens d'Eurojust qui lui sont nécessaires pour permettre l'accomplissement des missions du Réseau judiciaire européen. Dans la mesure où cela n'est pas incompatible avec l'autonomie fonctionnelle du secréta-riat du Réseau judiciaire européen, les règles s'appliquant aux membres du personnel d'Eurojust s'appliquent aux membres du secrétariat du Réseau judiciaire européen ;

c) les membres nationaux d'Eurojust peuvent participer aux réunions du Réseau judiciaire européen à l'invitation du Réseau judiciaire européen. Des points de contact du Réseau judiciaire européen peuvent, au cas par cas, être invités aux réunions d'Eurojust.

187 3. Eurojust établit et maintient une coopération étroite avec l'OLAF. À cette fin, l'OLAF peut contribuer aux travaux d'Euro-just visant à coordonner les enquêtes et poursuites en ce qui concerne la protection des intérêts financiers de la Commu-nauté, soit à l'initiative d'Eurojust, soit à la demande de l'OLAF, pour autant que les autorités nationales compétentes en la matière ne s'y opposent pas.

4. Pour les besoins de la réception et de la transmission des informations entre Eurojust et l'OLAF, et sans préjudice de l'article 9, les États membres veillent à ce que les membres nationaux d'Eurojust soient considérés comme des autorités compétentes des États membres pour les seuls besoins des règlements (CE) no 10731999 et (Euratom) n 10741999 du Conseil du 25 mai 1999 relatif aux enquêtes effectuées par l'Office européen de lutte antifraude (OLAF). L'échange d'in-formation entre l'OLAF et les membres nationaux est sans préjudice de l'information qui doit être fournie à d'autres auto-rités compétentes en vertu de ces règlements.

189 5. Eurojust peut établir des contacts et échanger des expé-riences de nature non opérationnelle avec d'autres instances, notamment des organisations internationales, en vue de l'ac-complissement de ses objectifs.

190 6. Eurojust peut, au cas par cas, coopérer avec des magis-trats de liaison des États membres, au sens de l'action commune 96/277/JAI du Conseil du 22 avril 1996 concernant un cadre d'échange de magistrats de liaison visant à l'améliora-tion de la coopération judiciaire entre les États membres de l'Union européenne.

191 Article 27

Échanges d'informations avec les partenaires 
192 1. Conformément à la présente décision, Eurojust peut échanger toute information nécessaire à l'accomplissement de ses tâches avec :

a) les instances compétentes en vertu des dispositions adoptées dans le cadre des traités ;

b) les organisations ou instances internationales ;

c) les autorités des États tiers compétentes pour les enquêtes et poursuites.

2. Avant tout échange d'informations entre Eurojust et les entités visées au paragraphe 1, points b) et c), le membre national de l'État membre qui a soumis les informations donne son accord au transfert de celles-ci. S'il y a lieu, le membre national consulte les autorités compétentes des États membres.

197 3. Eurojust peut conclure des accords de coopération, approuvés par le Conseil avec des États tiers et les entités visées au paragraphe 1. Ces accords peuvent notamment contenir des dispositions relatives à des arrangements pour le détachement d'officiers ou de magistrats de liaison auprès d'Eurojust. Ils peuvent également prévoir des dispositions concernant l'échange de données à caractère personnel. Dans ce cas, l'organe de contrôle commun est consulté par Eurojust.

198 Pour résoudre des questions urgentes, Eurojust peut également coopérer avec les entités visées au paragraphe 1 , points b) et c), sans conclure d'accord avec elles, à condition que cette coopé-ration n'implique pas la transmission par Eurojust de données à caractère personnel à ces entités.

199 4. Sans préjudice du paragraphe 3 , la transmission par Euro-just de données à caractère personnel aux entités visées au paragraphe 1 , point $b$ ), et aux autorités visées au paragraphe 1, point c), des États tiers qui ne sont pas soumis à l'application de la convention du Conseil de l'Europe du 28 janvier 1981 ne peut se faire que si un niveau suffisant comparable de protec-tion des données est assuré.

200 5. Si, par la suite, l'État tiers ou les entités visées au para-graphe 1, points b) et c), n'assurent pas le respect des condi-tions visées au paragraphe 4 ou qu'il y a de fortes raisons de penser qu'ils ne l'assurent pas, l'organe de contrôle commun et les États membres concernés sont immédiatement informés par Eurojust. L'organe de contrôle commun peut suspendre l'échange de données à caractère personnel avec les entités concernées jusqu'à ce qu'il ait constaté que des mesures ont été prises pour remédier à la situation.

201 6. Toutefois, même si les conditions visées aux paragraphes 3 et 4 ne sont pas réunies, un membre national, agissant en tant que tel, peut, à titre exceptionnel et uniquement pour que soient prises des mesures urgentes afin de prévenir un danger imminent et sérieux pour une personne ou la sécurité publique, procéder à un échange d'informations incluant des données à caractère personnel. C'est au membre national qu'il appartient de déterminer s'il est légal d'autoriser la communication. Il tient un relevé des communications de données qui ont été effec-tuées et des motifs pour lesquels elles l'ont été. La communica-tion de données n'est autorisée que si le destinataire s'engage à ce que les données ne soient utilisées qu'aux fins pour lesquelles elles ont été communiquées.

Article 28

Organisation et fonctionnement

203 1. Le collège est responsable de l'organisation et du fonc-tionnement d'Eurojust. 
204 2. Le collège élit un président parmi les membres nationaux et peut, s'il le juge nécessaire, élire deux vice-présidents au plus. Le résultat de cette élection est soumis au Conseil pour appro-bation.

3. Le président exerce sa fonction au nom du collège et sous son autorité, en conduit les travaux et contrôle la gestion quotidienne menée par le directeur administratif. Le règlement intérieur précise les cas où ses décisions ou actions doivent faire l'objet d'une autorisation préalable ou d'un rapport au collège.

4. La durée du mandat du président est de trois ans. Il peut être réélu une fois. La durée du mandat du (des) vice-prési-dent(s) éventuel(s) est régie par le règlement intérieur.

5. Eurojust est assisté par un secrétariat dirigé par un direc-teur administratif.

6. Eurojust exerce envers son personnel les pouvoirs dévolus à l'autorité investie du pouvoir de nomination. Le collège adopte les règles appropriées pour la mise en œuvre du présent paragraphe, conformément au règlement intérieur.

Article 29

Directeur administratif

210 1. Le directeur administratif d'Eurojust est nommé à l'unani-mité du collège. Le collège constitue un comité de sélection qui établit, après appel à candidature, une liste de candidats parmi lesquels le collège choisit le directeur administratif.

211 2. La durée du mandat du directeur administratif est de 5 ans. Ce mandat est renouvelable.

212 3. Le directeur administratif est soumis aux règlements et réglementations applicables aux fonctionnaires et autres agents des Communautés européennes.

213 4. Le directeur administratif travaille sous l'autorité du collège et de son président agissant conformément à l'article 28 , paragraphe 3 . Il peut être révoqué par le collège à la majorité des deux tiers.

214 5. Le directeur administratif est chargé de l'administration quotidienne d'Eurojust et de la gestion du personnel, sous le contrôle du président. (...)

215 Article 32

Information du Parlement européen et du Conseil

216 1. Le président, au nom du collège, rend compte au Conseil, par écrit et tous les ans, des activités et de la gestion, y compris budgétaire, d'Eurojust.

217 À cette fin, le collège prépare un rapport annuel sur les acti-vités d'Eurojust et sur les problèmes de politique criminelle au sein de l'Union qui auraient été mis en évidence à la suite des activités d'Eurojust. Dans ce rapport, Eurojust peut également formuler des propositions pour améliorer la coopération judi-ciaire en matière pénale.

218 Le président fournit également tout rapport ou toute autre information sur le fonctionnement d'Eurojust que le Conseil pourrait lui demander.

219 2. La présidence du Conseil adresse chaque année au Parle-ment européen un rapport sur les travaux menés par Eurojust ainsi que sur les activités de l'organe de contrôle commun. (...)

220 Article 41

Dispositions transitoires

221 1. Les membres nationaux de l'Unité provisoire de coopéra-tion judiciaire désignés par les États membres en vertu de la décision 2000/799/JAI du Conseil du 14 décembre 2000 
insti-tuant l'Unité provisoire de coopération judiciaire exercent la fonction de membre national d'Eurojust en vertu de l'article 2 de la présente décision jusqu'à la désignation définitive du membre national de l'État membre concerné et au plus tard jusqu'à l'expiration du deuxième mois qui suit la prise d'effet de la présente décision, date à laquelle cessent leurs fonctions.

À ce titre, les membres nationaux de l'Unité provisoire, jouissent de toutes les compétences qui sont celles des membres nationaux en vertu de la présente décision.

La désignation définitive du membre national prend effet au jour désigné à cet effet par l'État membre dans une notification au secrétariat général du Conseil par courrier officiel.

224 2. Un État membre peut déclarer, dans les trois mois qui suivent la prise d'effet de la présente décision, qu'il n'applique pas jusqu'à la date prévue à l'article 42 , certains articles, notam-ment les articles 9 et 13, au motif que cette application n'est pas compatible avec sa législation nationale. Le secrétariat général du Conseil informe les États membres et la Commission de cette déclaration.

3. Tant que le Conseil n'a pas approuvé le règlement inté-rieur d'Eurojust, le collège prend toutes ses décisions à la majorité des deux tiers, sauf lorsque la présente décision prévoit une décision à l'unanimité.

4. Les États membres veillent à ce que, jusqu'à la mise en place définitive d'Eurojust, toutes les mesures nécessaires soient prises afin de garantir que tous les dossiers traités par l'Unité provisoire de coopération judiciaire, notamment en matière de coordination des enquêtes et de poursuites, puissent continuer à être traités par les membres nationaux d'une manière efficace.

Les membres nationaux assurent au moins les mêmes objectifs et missions que l'Unité provisoire de coopération judiciaire.

Article 42

Transposition

es États membres mettent si nécessaire leur droit national en conformité avec la présente décision dans les meilleurs délais et en tout état de cause le 6 septembre 2003 au plus tard.

Article 43

Prise d'effet

ésente décision prend effet le jour de sa publication au journal officiel, sans préjudice de l'article 41. À partir de cette date, l'Unité provisoire de coopération judiciaire cesse d'exister. 
INDEX

Mots-clés : droit européen, textes et documents officiels

Thèmes : EUROJUST 